\title{
Quantitative theory of channeling particle diffusion in transverse energy in the presence of nuclear scattering and direct evaluation of dechanneling length
}

\author{
Victor V. Tikhomirov ${ }^{\mathrm{a}}$ \\ Institute for Nuclear Problems, Belarusian State University, Minsk, Belarus
}

Received: 20 January 2017 / Accepted: 12 July 2017 / Published online: 19 July 2017

(C) The Author(s) 2017. This article is an open access publication

\begin{abstract}
A refined equation for channeling particle diffusion in transverse energy taking into consideration largeangle scattering by nuclei is suggested. This equation is reduced to the Sturm-Liouville problem, allowing one to reveal both the origin and the limitations of the dechanneling length notion. The values of the latter are evaluated for both positively and negatively charged particles of various energies. New features of the dechanneling dynamics of positively charged particles are also revealed. First, it is demonstrated that the dechanneling length notion is completely inapplicable for their nuclear dechanneling process. Second, the effective electron dechanneling length of positively charged particle varies more than twice converging to a constant asymptotic value only at the depth exceeding the latter.
\end{abstract}

\section{Introduction}

Channeling effect in crystals delivers unique possibilities of both high energy charged particle radiation and control. Both electron and positron channeling makes it possible to devise new semi-monochromatic sources of $\mathrm{x}$ - and $\gamma$-radiation [13]. Proton and ion planar channeling in bent crystals is a promising tool for both extraction and collimation of the beams of the large hadron collider (LHC) and future circular collider (FCC) [4-6]. Planar channeling of charmed and beauty baryons in bent crystals also makes it possible to study the effects of both CP- and CPT violation [7].

All the applications of channeling are limited by its instability, induced by the dechanneling process. To describe the latter, the concept of particle diffusion in the energy of its transverse motion (transverse energy) was suggested soon after the channeling discovery [8-10].

\footnotetext{
a e-mail: vvtikh@mail.ru
}

The dechanneling process is often characterized by the visual notion of dechanneling length. Some information concerning the latter can be extracted from Monte Carlo simulations. However, since the average dechanneling distance of an individual particle strongly depends on its initial transverse energy, any direct method of dechanneling length evaluation through either the channeling fraction or dechanneling distance averaging over the incident particle angular distribution will give a result depending on the latter. In addition, there is no ground to expect that a channeling fraction, evaluated by any averaging method, will exponentially depend on the particle penetration depth, justifying an introduction of a dechanneling length independent of the latter.

In fact, only the theory of [10], describing collective properties of statistical particle behavior and consisting in finding the lowest eigen number of the diffusion equation, can be used for both strict introduction and evaluation of the dechanneling length. Since the dechanneling lengths of positively charged particles reach meters and tens of them at the LHC and FCC energies, the direct method [10] of their evaluation is more superior than the Monte Carlo simulations which become quite time consuming in the $\mathrm{TeV}$ particle energy region.

However, the theory [10] is applicable only in the case of electron dechanneling of non-relativistic ions. Since the critical channeling angle of low energy ions of mass $M$ considerably exceeds the maximal angle $\theta_{\max }=m / M$ of their scattering by electrons with mass $m$, the electron dechanneling of non-relativistic ions can be correctly described in neglect of both large-angle catastrophic scattering and the scattering angle fourth power contribution to the mean square variation of transverse energy. However, these assumptions lose their applicability in many other cases.

First of all, since the channeling angle decreases with energy, becoming much less than $\theta_{\max }=m / M$, the modification $[5,13]$ of the theory [10] for the ultrarelativistic case 
does not take into consideration adequately the particle scattering by crystal atom electrons. Also nuclear scattering both limits the fraction of channeling positively charged particles and is essential for channeling of negatively charged ones, considerably complicating the latter by the rechanneling process $[11,12]$.

Recently the experiments with bent crystals were first conducted to separate the nuclear dechanneling process of both positively $[14,15]$ and negatively [16] charged particles. The observed dechanneling fraction was fitted by the exponential decay law in order to introduce a constant nuclear dechanneling length. However, neither a justification of introduction nor a way of evaluation of the latter were suggested in [1416].

To provide a correct evaluation of the dechanneling length in the presence of nuclear scattering, a refined diffusion equation, which takes into consideration both the scattering angle fourth power contribution to the mean square variation of transverse energy and large-angle catastrophic scattering, is introduced in this paper. This equation is used to evaluate dechanneling length for the largest accelerator energies as well as to reveal the peculiarities of the dechanneling process introduced by both nuclear and electron scattering of both positively and negatively charged particles.

\section{Refined equation for channeled particle diffusion in transverse energy}

2.1 New features of the diffusion equation in transverse phase space

According to the Lindhard averaged potential concept $[1,5$, $8,9]$, particle motion at small angles with respect to crystal planes is described by the averaged atomic potential $V(x)$ (potential energy; see Fig. 1) whose translation symmetry justifies the introduction of the energy of transverse motion ${ }^{1}$

$\varepsilon_{\perp}=\varepsilon v_{x}^{2} / 2+V(x)=p_{x}^{2} / 2 \varepsilon+V(x)$,

or transverse energy for short, which is conserved and in which $\varepsilon$ is the total particle energy, $p_{x}=\varepsilon v_{x}$ and $v_{x}\left(\varepsilon_{\perp}, x\right)=\sqrt{2\left[\varepsilon_{\perp}-V(x)\right] / \varepsilon}$ are, respectively, its momentum and velocity projections on the $x$ axis, which is normal to the crystal planes. Conservative particle motion in the potential $V(x)$ is always disturbed by the incoherent scattering by both nuclei and electrons - see Fig. 1. At that, an instant incoherent deflection by the angle $\theta_{x}$ in the point $x$ induces a transverse energy change from (1) to

$\varepsilon_{\perp}^{\prime}=\varepsilon\left[v_{x}(x)+\theta_{x}\right]^{2} / 2+V(x)=\varepsilon_{\perp}+\varepsilon v_{x}(x) \theta_{x}+\varepsilon \theta_{x}^{2} / 2$.

${ }^{1}$ The system of units $\hbar=c=1$ is used.
To describe the cumulative result of such changes, FokkerPlanck approximation $[1,5,17,18]$, which is able to treat small transverse energy changes only $[5,10,18]$, is widely applied. Following the well established procedure [17] and using the notations of [18], one can introduce a distribution function in the one-dimensional transverse phase space

$F\left(\varepsilon_{\perp}, x, z\right)=\varphi\left(\varepsilon_{\perp}, z\right) f_{\varepsilon_{\perp}}(x)$

and the same over the transverse energy

$\varphi\left(\varepsilon_{\perp}, z\right) \equiv \frac{1}{N} \frac{\mathrm{d} N}{\mathrm{~d} \varepsilon_{\perp}}$

where

$f_{\varepsilon_{\perp}}(x)=\frac{2}{T v_{x}\left(\varepsilon_{\perp}, x\right)}$

is the coordinate space distribution function in which

$T\left(\varepsilon_{\perp}\right)=2 \int_{x_{l}\left(\varepsilon_{\perp}\right)}^{x_{r}\left(\varepsilon_{\perp}\right)} \frac{\mathrm{d} x}{v_{x}\left(\varepsilon_{\perp}, x\right)}$

$x_{l}\left(\varepsilon_{\perp}\right)$ and $x_{r}\left(\varepsilon_{\perp}\right)$ are the period, the right and the left turning points of the channeling motion at given $\varepsilon_{\perp}$. The dependence of the phase space distribution function (3) on the depth $z$ of particle penetration into the crystal along the channeling planes is governed by the equation

$\frac{\partial F}{\partial z}=-\frac{\partial}{\partial \varepsilon_{\perp}}\left(\frac{\Delta \varepsilon_{\perp}}{\Delta z} F\right)+\frac{1}{2} \frac{\partial^{2}}{\partial \varepsilon_{\perp}^{2}}\left(\frac{\left(\Delta \varepsilon_{\perp}\right)^{2}}{\Delta z} F\right)-w F$

$\frac{\Delta \varepsilon_{\perp}}{\Delta z}=\frac{\Delta \varepsilon_{\perp}\left(\varepsilon_{\perp}, x\right)}{\Delta z}=\int\left(\varepsilon_{\perp}^{\prime}-\varepsilon_{\perp}\right) \mathrm{d} \Sigma$,

$\frac{\left(\Delta \varepsilon_{\perp}\right)^{2}}{\Delta z}=\frac{\left(\Delta \varepsilon_{\perp}\right)^{2}\left(\varepsilon_{\perp}, x\right)}{\Delta z}=\int\left(\varepsilon^{\prime} \perp-\varepsilon_{\perp}\right)^{2} \mathrm{~d} \Sigma$,

which was deduced following [18] and supplemented here for the first time by the last term, which contains the probability

$w=w\left(\varepsilon_{\perp}, x\right)=\int^{\prime} \mathrm{d} \Sigma$

of "catastrophic" scattering and describes the single scattering process of particle immediate knocking out from the channeling state. Another new feature will be the preservation of the fourth scattering angle power contribution to the integrand of Eq. (9); to introduce it a specific definition of the integral regions of Eqs. (8)-(10) is introduced below.

To make the consideration more transparent, we will use a simplified expression for the particle macroscopic scattering 


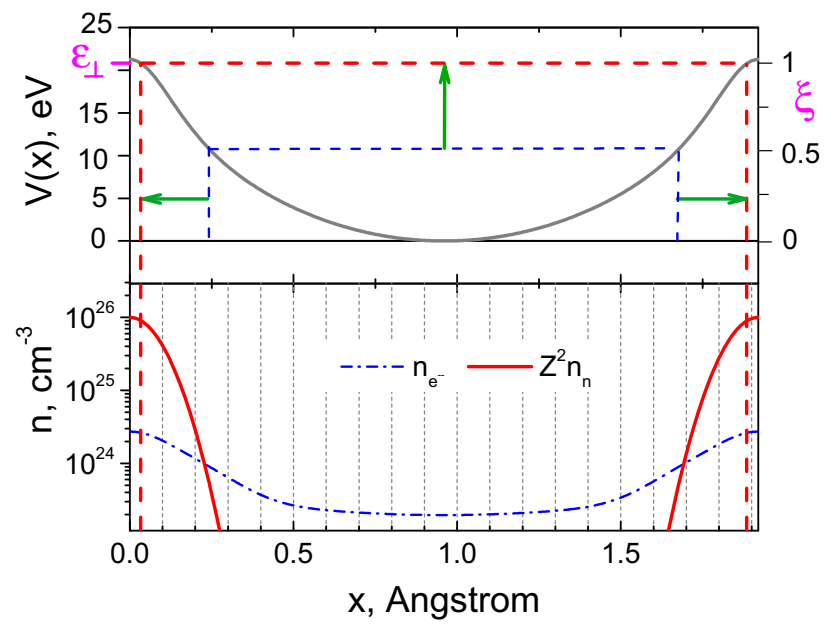

Fig. 1 Planar potential and the undertaken expansion of the considered region of positively charged particle motion, shown by arrows (top). Averaged number density of electrons and the same, multiplied by the atomic number squared, of nuclei (bottom)

cross section on both nuclei with local number density $n_{\mathrm{n}}(x)$ and electrons with local number density $n_{\mathrm{e}}(x)$ :

$\mathrm{d} \Sigma=\frac{4 \alpha^{2}\left[Z^{2} n_{\mathrm{n}}(x)+n_{\mathrm{e}}(x)\right]}{\beta^{2} p^{2}\left(\theta^{2}+\theta_{1}^{2}\right)^{2}} \mathrm{~d} \theta_{x} \mathrm{~d} \theta_{y}$,

where $\theta_{1}$ is the angle, which takes the atomic nucleus potential screening into consideration. Both $Z^{2} n_{\mathrm{n}}(x)$ and $n_{\mathrm{e}}(x)$ coordinate dependence is illustrated by Fig. 1 . The integrand of the mean-squared transverse energy variation rate (9),

$\left(\varepsilon^{\prime} \perp-\varepsilon_{\perp}\right)^{2}=2 \varepsilon\left[\varepsilon_{\perp}-V(x)\right] \theta_{x}^{2}+\varepsilon^{2} \theta_{x}^{4} / 4+\cdots$,

in which the odd powers of the scattering angle $\theta_{x}$ are omitted, contains the fourth power contribution of the same, which has never been taken into consideration before [5,10,13]. The point is that, as can be directly seen from Eqs. (11) and (12), this contribution diverges at large $\theta_{x}$ and to treat it, finite integration limits should be introduced. To define them, the transverse energy (2) of the scattered particle has been equated to the height $V_{\max }$ of the planar potential. The boundary scattering angles which then follow,

$\theta_{ \pm}\left(\varepsilon_{\perp}, x\right)=-v_{x}\left(\varepsilon_{\perp}, x\right) \pm \sqrt{2\left(V_{\max }-V(x)\right) / \varepsilon}$,

limit the integration region in Eq. (8) and (9) by the angles $\theta_{-}\left(\varepsilon_{\perp}, x\right) \leq \theta_{x} \leq \theta_{+}\left(\varepsilon_{\perp}, x\right)$, leave the channeling particles channeled, and fix the complementary integration regions $\theta_{x}>\theta_{+}\left(\varepsilon_{\perp}, x\right), \theta_{x}<\theta_{-}\left(\varepsilon_{\perp}, x\right)$ in Eq. (10). The integration over all the possible angles $\theta_{y}=\sqrt{\theta^{2}-\theta_{x}^{2}}$ of scattering in the $y z$ plane is assumed everywhere in Eqs. (8)-(10).
Given the integration limits explicitly determined, the integrals (8)-(10) can be routinely taken with the result

$$
\begin{aligned}
\frac{\Delta \varepsilon_{\perp}\left(\varepsilon_{\perp}, x\right)}{\Delta z}= & \frac{\pi \alpha^{2}}{\beta^{3} p}\left[Z^{2} n_{\mathrm{n}}(x)+n_{\mathrm{e}}(x)\right] \\
& \times\left\{\ln \left[\frac{\theta_{+}(x)+\sqrt{\theta_{+}^{2}(x)+\theta_{1}^{2}}}{\theta_{-}(x)+\sqrt{\theta_{-}^{2}(x)+\theta_{1}^{2}}}\right]\right. \\
& \left.+\frac{\theta_{-}(x)}{\sqrt{\theta_{-}^{2}(x)+\theta_{1}^{2}}}-\frac{\theta_{+}(x)}{\sqrt{\theta_{+}^{2}(x)+\theta_{1}^{2}}}\right\}
\end{aligned}
$$

for the rate of transverse energy variation growth;

$$
\frac{\left(\Delta \varepsilon_{\perp}\right)^{2}\left(\varepsilon_{\perp}, x\right)}{\Delta z}=a\left(\varepsilon_{\perp}, x\right)+b\left(\varepsilon_{\perp}, x\right),
$$

where

$a\left(\varepsilon_{\perp}, x\right)=4\left[\varepsilon_{\perp}-V(x)\right] \frac{\Delta \varepsilon_{\perp}}{\Delta z}$

and

$$
\begin{aligned}
b\left(\varepsilon_{\perp}, x\right)= & \frac{\pi \alpha^{2}}{4}\left[Z^{2} n_{\mathrm{n}}(x)+n_{\mathrm{e}}(x)\right] \\
& \times\left\{\theta_{+}(x) \sqrt{\theta_{+}^{2}(x)+\theta_{1}^{2}}-\theta_{-}(x) \sqrt{\theta_{-}^{2}(x)+\theta_{1}^{2}}\right. \\
& +\frac{2 \theta_{1}^{2} \theta_{+}(x)}{\sqrt{\theta_{+}^{2}(x)+\theta_{1}^{2}}}-\frac{2 \theta_{1}^{2} \theta_{-}(x)}{\sqrt{\theta_{-}^{2}(x)+\theta_{1}^{2}}} \\
& \left.-3 \theta_{1}^{2} \ln \left[\frac{\theta_{+}(x)+\sqrt{\theta_{+}^{2}(x)+\theta_{1}^{2}}}{\theta_{-}(x)+\sqrt{\theta_{-}^{2}(x)+\theta_{1}^{2}}}\right]\right\}
\end{aligned}
$$

for the rate of squared transverse energy variation growth and

$$
\begin{aligned}
w\left(\varepsilon_{\perp}, x\right)= & \frac{\pi \alpha^{2}}{\beta^{2} p^{2} \theta_{1}^{2}}\left[Z^{2} n_{\mathrm{n}}(x)+n_{\mathrm{e}}(x)\right] \\
& \times\left\{2+\frac{\theta_{-}(x)}{\sqrt{\theta_{-}^{2}(x)+\theta_{1}^{2}}}-\frac{\theta_{+}(x)}{\sqrt{\theta_{+}^{2}(x)+\theta_{1}^{2}}}\right\}
\end{aligned}
$$

for the catastrophic scattering probability.

\subsection{Reduction of the diffusion equation to the transverse energy space}

To reduce the diffusion equation (7) in the transverse phase space to that in the transverse energy space, the averaging over the period of transverse motion

$$
\left\langle\Phi\left(\varepsilon_{\perp}, x\right)\right\rangle=\int_{x_{l}\left(\varepsilon_{\perp}\right)}^{x_{r}\left(\varepsilon_{\perp}\right)} \Phi\left(\varepsilon_{\perp}, x\right) f_{\varepsilon_{\perp}}(x) \mathrm{d} x
$$

is used [18] resulting in the equation 


$$
\begin{aligned}
\frac{\partial \varphi\left(\varepsilon_{\perp}, z\right)}{\partial z}= & -\frac{\partial}{\partial \varepsilon_{\perp}}\left(A\left(\varepsilon_{\perp}\right) \frac{\partial}{\partial \varepsilon_{\perp}} \frac{\varphi\left(\varepsilon_{\perp}, z\right)}{T\left(\varepsilon_{\perp}\right)}\right) \\
& +\frac{\partial^{2}}{\partial \varepsilon_{\perp}^{2}}\left(B\left(\varepsilon_{\perp}\right) \frac{\varphi\left(\varepsilon_{\perp}, z\right)}{T\left(\varepsilon_{\perp}\right)}\right)-W\left(\varepsilon_{\perp}\right) \varphi\left(\varepsilon_{\perp}, z\right),
\end{aligned}
$$

containing the averaged coefficients

$$
\begin{aligned}
A\left(\varepsilon_{\perp}\right) & =\left\langle\frac{\Delta \varepsilon_{\perp}\left(\varepsilon_{\perp}, x\right)}{\Delta z}\right\rangle, \\
B\left(\varepsilon_{\perp}\right) & =\left\langle b\left(\varepsilon_{\perp}, x\right)\right\rangle, \\
W\left(\varepsilon_{\perp}\right) & =\left\langle w\left(\varepsilon_{\perp}, x\right)\right\rangle .
\end{aligned}
$$

Equation (20) suggests, instead of transverse energy, to introduce both the variable

$\xi^{\prime}\left(\varepsilon_{\perp}\right)=\int_{0}^{\varepsilon_{\perp}} T\left(\varepsilon_{\perp}\right) \mathrm{d} \varepsilon_{\perp}$

and the corresponding distribution function

$u(\xi)=\frac{\varphi\left(\varepsilon_{\perp}\right)}{T\left(\varepsilon_{\perp}\right)}=\frac{1}{N} \frac{\mathrm{d} N}{T\left(\varepsilon_{\perp}\right) d \varepsilon_{\perp}}=\frac{1}{N} \frac{\mathrm{d} N}{\mathrm{~d} \xi^{\prime}}$.

The variable (22) has a clear semiclassical interpretation, being equal to the quantum number of transverse oscillatory motion in the quantum state corresponding to the considered transverse energy $\varepsilon_{\perp}$ multiplied by $2 \pi$. Both Eqs. (22), (23) and the standard transformations [19] allow one to extract further the Sturm-Liouville operator in Eq. (20)

$r\left(\xi^{\prime}\right) \frac{\partial u\left(\xi^{\prime}, z\right)}{\partial z}=\frac{\partial}{\partial \xi^{\prime}}\left(p^{\prime}\left(\xi^{\prime}\right) \frac{\partial u\left(\xi^{\prime}, z\right)}{\partial \xi^{\prime}}\right)-q\left(\xi^{\prime}\right) u\left(\xi^{\prime}, z\right)$

with the coefficients

$$
\begin{aligned}
& p^{\prime}\left(\xi^{\prime}\right)=\left[B\left(\varepsilon_{\perp}\left(\xi^{\prime}\right)\right)+A\left(\varepsilon_{\perp}\left(\xi^{\prime}\right)\right)\right] T\left(\varepsilon_{\perp}\left(\xi^{\prime}\right)\right) r\left(\xi^{\prime}\right), \\
& q\left(\xi^{\prime}\right)=\left[W\left(\varepsilon_{\perp}\left(\xi^{\prime}\right)\right)-B^{\prime \prime}\left(\varepsilon_{\perp}\left(\xi^{\prime}\right)\right)\right] T^{-1}\left(\varepsilon_{\perp}\left(\xi^{\prime}\right)\right) r\left(\xi^{\prime}\right),
\end{aligned}
$$

and

$$
\begin{aligned}
r\left(\xi^{\prime}\right) & =\exp \int_{0}^{\varepsilon_{\perp}\left(\xi^{\prime}\right)} \frac{B^{\prime}\left(\varepsilon_{\perp}\right) \mathrm{d} \varepsilon_{\perp}}{A\left(\varepsilon_{\perp}\right)+B\left(\varepsilon_{\perp}\right)}, \\
\varepsilon_{\perp}\left(\xi^{\prime}\right) & =\int_{0}^{\xi^{\prime}} \frac{\mathrm{d} \xi}{T\left(\varepsilon_{\perp}(\xi)\right)},
\end{aligned}
$$

which can be readily calculated using Eqs. (14)-(19) and (21). It should be mentioned that Eq. (24) is more general than that used in [10,13], which follows from Eq. (24) at $q=0$ and $r=$ const.
Table 1 Dechanneling length and precision of its evaluation

\begin{tabular}{llll}
\hline Potential & $\frac{\delta \varepsilon_{\perp}\left(\varepsilon_{\perp \max }\right)}{V_{\max }-\varepsilon_{\perp \max }}$ & $l_{\text {dech }}, \mathrm{cm}$ & $\Delta l_{\text {dech }}, \%$ \\
\hline Tob [21] & 1 & 23.1 & 0 \\
Tob [21] & 0.5 & 22.9 & -0.81 \\
Tob [21] & 2 & 23.2 & +0.37 \\
DT [22] & 1 & 23.3 & +0.61 \\
Mol [9] & 1 & 21.4 & -7.255 \\
\hline
\end{tabular}

\subsection{Diffusion equation boundary conditions}

Introducing the boundary conditions for Eq. (24), we immediately adopt the one of $\partial u(0) / \partial \xi^{\prime}=0$, reflecting the impossibility of both transverse energy and variable (22) to drop below zero. However, another condition of the distribution function (23) nullification at some $\varepsilon_{\perp \text { max }}$ or $\xi_{\text {max }}^{\prime}$, essential for the present approach $[10,13]$, needs some comments. Indeed, at first glance, the region of large $\varepsilon_{\perp}$ is surely well populated by the intensively scattering dechanneling particles. However, the diffusion equation approach is in general applicable in the limit of small changes of the considered quantity, transverse energy in our case. That is why one should adopt the idea that at some $\varepsilon_{\perp}^{\prime}$ or $\xi_{\max }^{\prime}$, when the variation

$$
\begin{aligned}
\delta \varepsilon_{\perp}\left(\varepsilon^{\prime}\right)= & \left(\left\langle\frac{\left(\Delta \varepsilon_{\perp}\right)^{2}\left(\varepsilon_{\perp}^{\prime}, x\right)}{\Delta z}\right\rangle T\left(\varepsilon^{\prime}\right)\right. \\
& \left.-\left\langle\frac{\Delta \varepsilon_{\perp}\left(\varepsilon_{\perp}^{\prime}, x\right)}{\Delta z}\right\rangle^{2} T^{2}\left(\varepsilon_{\perp}^{\prime}\right)\right)^{1 / 2}
\end{aligned}
$$

of the former over the channeling period reaches the interval $V_{\text {max }}-\varepsilon_{\perp}^{\prime}$, separating $\varepsilon_{\perp}^{\prime}$ from the potential maximum, the diffusion equation (24) ceases to describe any particle, justifying thus the second boundary condition $u\left(\xi_{\max }^{\prime}\right)=0$, where $\xi_{\max }^{\prime}=\xi^{\prime}\left(\varepsilon_{\perp \max }\right)$ and $\delta \varepsilon_{\perp}\left(\varepsilon_{\perp \max }\right)=V_{\max }-\varepsilon_{\perp \max }$. To estimate the uncertainly of the latter definition of $\varepsilon_{\perp \max }$, we took the ratios

$\delta \varepsilon_{\perp}\left(\varepsilon_{\perp \max }\right) /\left[V_{\max }-\varepsilon_{\perp \max }\right]=0.5,1,2$

to demonstrate in Table 1 that the uncertainty of the dechanneling length definition is marginal.

Given the boundary value $\xi_{\max }^{\prime}$, one can now redefine Eq. (24) in order to formulate Sturm-Liouville problem on the interval $[0,1]$ of the normalized variable $\xi=\xi^{\prime} / \xi_{\max }^{\prime}$ by omitting the prime in Eqs. (23)-(27) and putting $p^{\prime}(\xi)=$ $p(\xi) \xi_{\max }^{\prime 2}$ :

$r(\xi) \frac{\partial u(\xi, z)}{\partial z}=\frac{\partial}{\partial \xi}\left(p(\xi) \frac{\partial u(\xi, z)}{\partial \xi}\right)-q(\xi) u(\xi, z)$, 


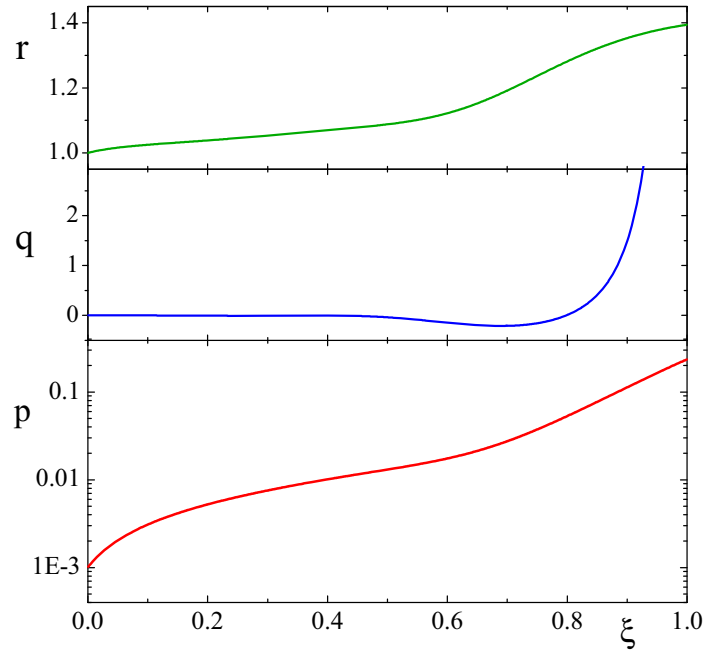

Fig. 2 Equations (29) and (30) as regards coefficient dependence on the parameter $\xi$ for $400 \mathrm{GeV}$ protons channeled by (110) Si planes

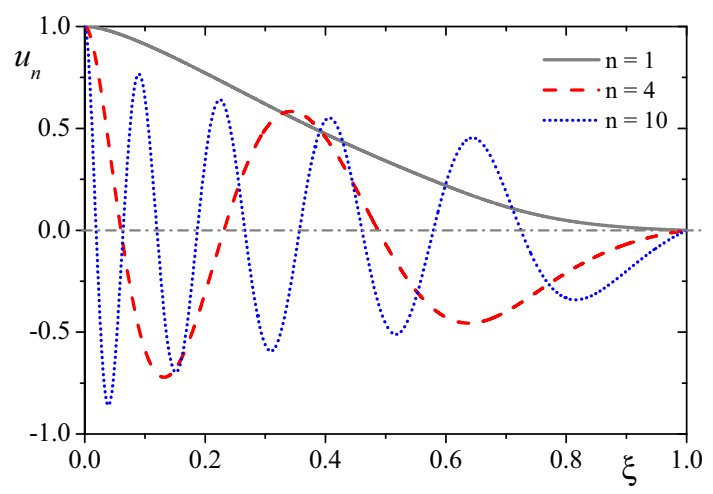

Fig. 3 The first, fourth and tenth eigen states for the same

$-\frac{\partial}{\partial \xi}\left[p(\xi) \frac{\partial}{\partial \xi} u_{n}(\xi)\right]+q(\xi) u_{n}(\xi)=\lambda_{n} r(\xi) u_{n}(\xi)$,

$\partial u_{n}(0) / \partial \xi=0, \quad u_{n}(1)=0, \quad n=1,2, \ldots$,

where we follow the sign convention of [20]. The dependence on the normalized parameter $\xi$ of Eqs. (29), (30) as regards the coefficients is illustrated by Fig. 2 in the case of $400 \mathrm{GeV}$ protons and (110) Si plane, also used as an example in Figs. $3,4,5,6$ and 7 .

\section{Diffusion equation solution and its analysis}

\subsection{Dechanneling length at different energies}

A numerical solution of Sturm-Liouville problem (30), (31), like that of [20], allows one to find any number of its eigen states $u_{n}(\xi)$ and eigen values $\lambda_{n}, n=1,2, \ldots$, some of the lower of which are plotted, respectively, in Figs. 3 and 4.

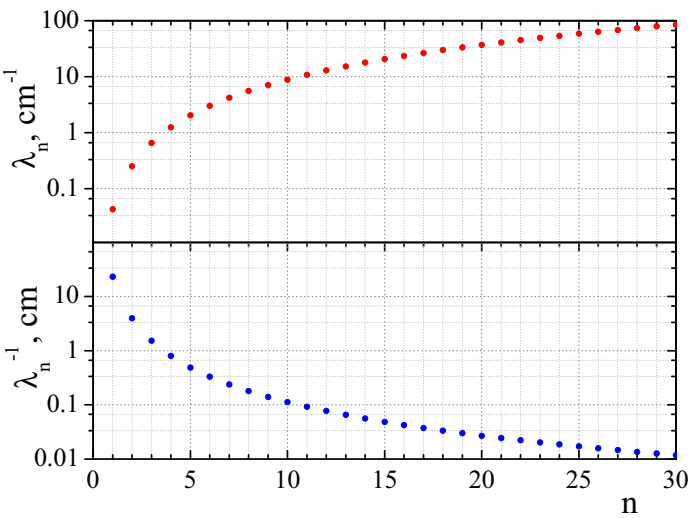

Fig. 4 The eigen values of Eq. (30) (top) and corresponding decay lengths (bottom)

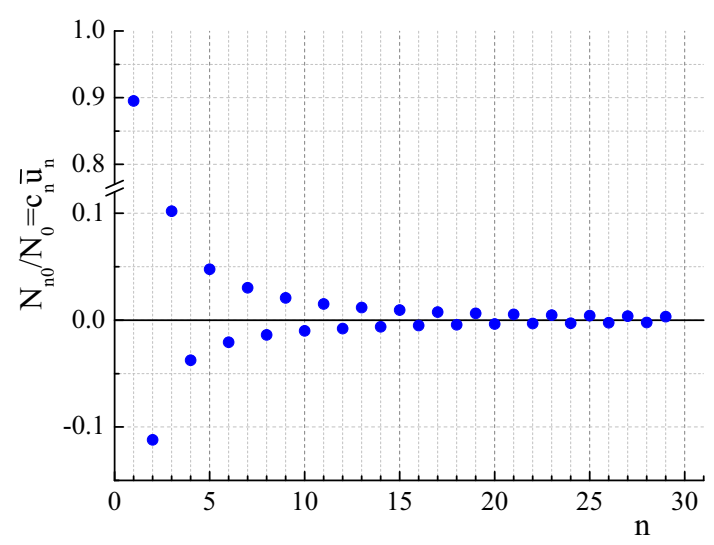

Fig. 5 The eigen state amplitudes entering Eq. (33)

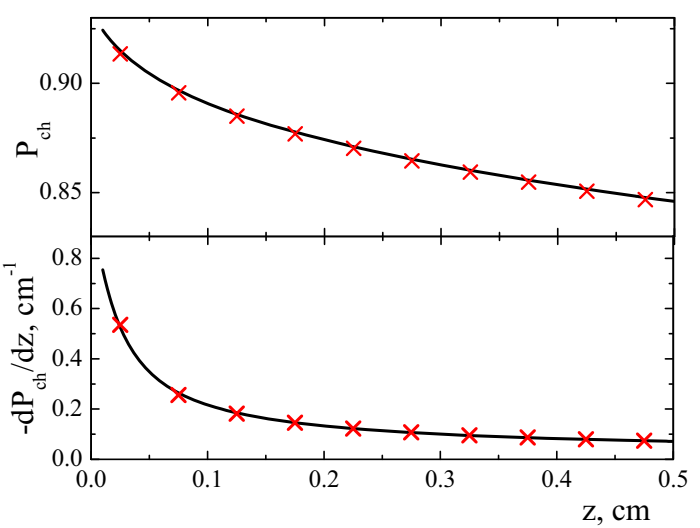

Fig. 6 Crystal depth dependence of the channeling probability (33) (solid line, top) and dechanneling rate (solid line, bottom) and their fits by the function (37) and its reversed-sign derivative, respectively (crosses)

The eigen values $\lambda_{n}$ are positive and monotonically increase with their number $n$, equal to that of the corresponding eigen state $u_{n}(\xi)$ nodes minus one. The completeness property of the system of eigen states of the problem (30), (31) allows one to represent any solution of Eq. (29) in the form of an expansion, 


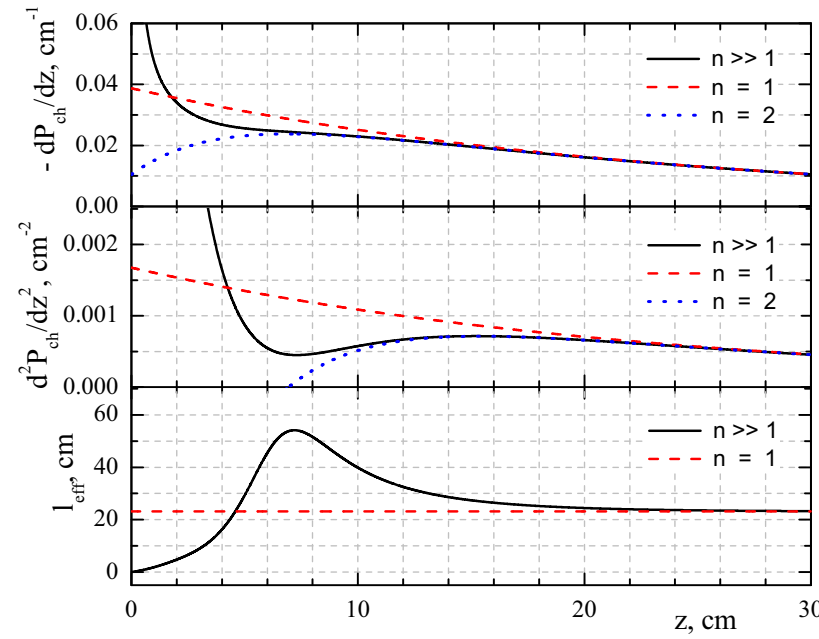

Fig. 7 Dechanneling rate (38), its derivative (39) and effective dechanneling length (40) versus crystal depth

$u(\xi, z)=\sum_{n=1}^{\infty} c_{n} \exp \left(-\lambda_{n} z\right) u_{n}(\xi)$,

the coefficients $c_{n}$ of which are determined by the distribution $u(\xi, 0)$ at the crystal entrance $z=0$ and are evaluated below. The solution (32) allows one to determine the channeling probability dependence on crystal depth

$$
\begin{aligned}
P_{\mathrm{ch}}(z) & \equiv \frac{N_{c h}(z)}{N_{0}}=\int_{0}^{1} u(\xi, z) \mathrm{d} \xi=\frac{1}{N_{0}} \sum_{n=1}^{\infty} N_{n 0} \exp \left(-\lambda_{n} z\right) \\
& =\sum_{n=1}^{\infty} c_{n} \bar{u}_{n} \exp \left(-\lambda_{n} z\right) \stackrel{z \lambda_{1}>1}{\longrightarrow} c_{1} \bar{u}_{1} \exp \left(-\lambda_{1} z\right),
\end{aligned}
$$

where

$\bar{u}_{n}=\int_{0}^{1} u_{n}(\xi) \mathrm{d} \xi$

An exponential decay of the eigen states is governed by their eigen values, the smallest first of which determines alone the asymptotic exponential behavior of the general solution (32), which gave rise to the introduction of the dechanneling length $l_{\mathrm{dech}}=1 / \lambda_{1}$ in [10]. Uncertainties of the latter, connected with both the qualitative nature of the introduction of the boundary $\varepsilon_{\perp \max }$ of the diffusion approximation applicability region and the planar potential model, are displayed in Table 1 for $400 \mathrm{GeV}$ protons and (110) Si planes. As one can see, both the uncertainty of the boundary energy $\varepsilon_{\perp \text { max }}$ definition and the transition between the potential models [21,22] change the $l_{\text {dech }}$ value by less than one percent. A considerably smaller dechanneling length, obtained with the Molière potential [9], reflects mainly the limited applicability of the
Table 2 Dechanneling lengths for protons and electrons of different energies

\begin{tabular}{lllccl}
\hline$e^{-} / p$ & $\varepsilon, \mathrm{GeV}$ & $l_{\mathrm{dech}}, \mathrm{cm}$ & $\lambda_{2} / \lambda_{1}$ & $\Delta l_{\mathrm{dech}}, \%$ & $N_{\mathrm{ch} 0} / N_{0}$ \\
\hline$p$ & 400 & 23.1 & 6.0 & 0.61 & 0.895 \\
$p$ & 6500 & 303.6 & 5.7 & 0.34 & 0.895 \\
$p$ & $10^{5}$ & 3936.0 & 5.6 & 0.18 & 0.895 \\
$e^{-}$ & 1 & $6.0 \times 10^{-4}$ & 7.8 & 130.0 & 0.33 \\
$e^{-}$ & 10 & $50.0 \times 10^{-4}$ & 6.9 & 78.0 & 0.39 \\
$e^{-}$ & 100 & 0.044 & 6.4 & 46.0 & 0.44 \\
$e^{-}$ & 1000 & 0.38 & 6.1 & 28.5 & 0.49 \\
\hline
\end{tabular}

latter to the channeling phenomenon. In any case, the precision of $l_{\mathrm{dech}}$ determination by the diffusion equation method is not worse than the uncertainty related with the potential model choice.

Table 2 displays the $l_{\text {dech }}$ values for both positively and negatively charged ultra-relativistic particles demonstrating the large value of the ratio $\lambda_{2} / \lambda_{1} \sim 6 \div 8$, assuring the strong dominance of the first eigen state starting from $z \sim l_{\text {dech }}$ (see below).

\subsection{Nuclear dechanneling probability dependence on particle penetration depth}

However, the evolution of the probability (33) at the smaller depths $z \leq l_{\text {dech }}$ is governed by many states, making its behavior more complex. To study an interference of different eigen states, one should know their amplitudes $N_{n 0} / N_{0}=$ $c_{n} \bar{u}_{n}$ (see Eq. (33)). The simplest formula can be obtained for them in the limit of zero-angle particle incidence, when $\left|\mathrm{d} \varepsilon_{\perp}\right|=|\mathrm{d} V(x) / \mathrm{d} x| \mathrm{d} x$ and the distribution function at the crystal entrance reduces to

$u(\xi, 0)=\frac{1}{N} \frac{\mathrm{d} N}{\mathrm{~d} \xi}=\frac{\xi_{\max }^{\prime}}{d_{\mathrm{pl}} T\left(\varepsilon_{\perp}(\xi)\right)\left|\mathrm{d} V\left(x\left(\varepsilon_{\perp}(\xi)\right)\right) / \mathrm{d} x\right|}$,

where $d_{\mathrm{pl}}$ is the inter-planar distance, allowing one to evaluate both the coefficients

$$
\begin{aligned}
c_{n} & =\int_{0}^{1} u(\xi, 0) u_{n}(\xi) r(\xi) \mathrm{d} \xi\left(\int_{0}^{1} u_{n}^{2}(\xi) r(\xi) \mathrm{d} \xi\right)^{-1} \\
& =\int_{0}^{d} u_{n}(\xi(V(x))) r(\xi(V(x))) \frac{\mathrm{d} x}{d}\left(\int_{0}^{1} u_{n}^{2}(\xi) r(\xi) \mathrm{d} \xi\right)^{-1}
\end{aligned}
$$

of the expansion (32) and the amplitudes $N_{n 0} / N_{0}=c_{n} \bar{u}_{n}$ of the eigen states, which enter Eq. (33) and are plotted in Fig. 5. 
Equations (33) and (36) allow one to illustrate the main features of the dechanneling process of positively charged particles in the most practically important and complex region $l<l_{\text {dech. }}$. Since only the particles with sufficiently high transverse energies reach the regions of atomic nuclei localization (see Fig. 1), the fast nuclear and slow electron dechanneling processes, which dominate, respectively, at sufficiently high and low transverse energies, can be qualitatively distinguished. A question arises, however: is it possible to strictly introduce and measure both the nuclear and the electron dechanneling lengths, as was done in $[14,15]$ ? It is the knowledge of both the eigen values and the amplitudes (36) of the eigen states, entering the solution (32), which makes it possible to treat this question thoroughly.

Figure 4 shows that the number of the eigen values, the inverse values of which correspond to the typical nuclear dechanneling region (about $1 \mathrm{~mm}$ at $400 \mathrm{GeV}$ ), exceeds one considerably. Corresponding eigen states also have the amplitudes, comparable in value. The cumulative contribution of these multiple and close fast decaying states is approximated by the integral of the exponential function $\exp \left(-\lambda\left(\varepsilon_{\perp}\right) z\right)$ product by a slowly varying function of transverse energy, well fitted by the power-type

$P_{\mathrm{ch}}(z)=\sum_{n=1}^{\infty} c_{n} \bar{u}_{n} \exp \left(-\lambda_{n} z\right) \stackrel{z \ll \lambda_{1}}{\longrightarrow} 0.954-0.137 \sqrt[3]{z}$,

instead of exponential-type function of the crystal depth $z$, as Fig. 6 demonstrates. Thus, in place of the dechanneling length extraction, the experiments on nuclear dechanneling should better accept the power-type fitting of the channeling fraction dependence on depth.

\subsection{Peculiarities of the electron dechanneling of positively charged particles}

On the opposite, a few lowest eigen values, describing the electron dechanneling process, differ severalfold-see Fig. 4. In addition, the smallest of them possesses an extraordinary large amplitude $N_{10} / N_{0}=c_{1} \bar{u}_{1}$-see Fig. 5. By this reason the applicability of the electron dechanneling length notion is ensured starting just from $z \simeq l_{\text {dech }}=1 / \lambda_{1}$. However, the real experiments on beam steering, collimation and electromagnetic radiation generation are conducted at $z<l_{\text {dech }}$ and even at $z \ll l_{\text {dech }}$, where several lowest eigen state contribute considerably to both Eqs. (32) and (33), violating their asymptotic exponential behavior. Indeed, let us consider both the dechanneling rate

$$
-P_{\mathrm{ch}}^{\prime}(z)=\sum_{n=1}^{\infty} c_{n} \bar{u}_{n} \lambda_{n} \exp \left(-\lambda_{n} z\right),
$$

which can be measured experimentally, and its derivative
$P_{\mathrm{ch}}^{\prime \prime}(z)=\sum_{n=1}^{\infty} c_{n} \bar{u}_{n} \lambda_{n}^{2} \exp \left(-\lambda_{n} z\right)$

(see Fig. 7). The point is that the relatively small population coefficients $\left|c_{n}\right| \ll c_{1}$ with $n>1$ are multiplied by the large eigen values $\lambda_{n} \gg \lambda_{1}$ and their squares in Eqs. (38) and (39), respectively, making the "weights" of the states with $n>1$ comparable with that of the first one. Having, in addition to its high "weight", the negative sign of the amplitude, the second eigen state directly distorts the steady decrease of the leading contribution of the first one. To demonstrate at what a degree the states with $n>1$ modify the exponential channeling decay law at $z<l_{\text {dech }}$, one should consider the effective dechanneling length

$l_{\text {dech }}^{\mathrm{eff}}(z)=-\frac{P_{c h}^{\prime}(z)}{P_{c h}^{\prime \prime}(z)}$.

The latter equals the constant value $l_{\text {dech }}=1 / \lambda_{1}$ either when only the first terms in the sums (38) and (39) are preserved or if the region $z \gg l_{\text {dech }}$ is considered. However, at $z<l_{\text {dech }}$ the $n>1$ terms induce a considerable dependence of the effective dechanneling length (40) on depth, resulting in its doubling at $z=6 \div 8 \mathrm{~cm}$-see Fig. 7 .

These limitations of the region of dechanneling length notion applicability should be taken for granted. First of all, the possibility to introduce dechanneling length itself is nontrivial and was impossible without serious mathematical treatment. The latter has demonstrated that the exponential decay of channeling fraction indeed is established at sufficiently large crystal depth, becoming possible due to the existence of a unique transverse energy distribution $u_{1}\left(\xi\left(\varepsilon_{\perp}\right)\right)$, which preserves its shape, decreasing proportionally to the exponential $\exp \left(-z / l_{\text {dech }}\right)$ at any $\varepsilon_{\perp}$. In principle, if the initial distribution $u(\xi, 0)$ coincided with $u_{1}(\xi)$, an exponential decay could be observed starting from $z=0$. However, since, in fact, $u(\xi, 0)$ considerably differs from $u_{1}(\xi)$ at various incident particle angular spreads, some transformation of the former to the latter has to occur before the exponential decay is established. This transformation is described as a decay of the eigenstates $u_{n}(\xi)$ with $n>1$. In particular, the ones with $\lambda_{n} \gg 1 / l_{\text {dech }}$ describe fast nuclear dechanneling process which originates from the upper underbarrier transverse energy region and is illustrated by Fig. 6. It was demonstrated that both the large number and the nearness of the eigen values ensure the power-law nuclear dechanneling process, instead of the exponential one, at $z \ll l_{\text {dech. }}$.

However, an arbitrary initial distribution $u(\xi, 0)$ differs from $u_{1}(\xi)$ in the deep under-barrier region as well. The termination of the $u(\xi, 0)$ relaxation into the quasistationary state $u_{1}(\xi)$ in the latter is described by only a few lowest eigenstates. Since any eigenstate simultaneously describes 
both the deep under-barrier and the upper near-barrier channeling particles (see Fig. 3), any redistribution in the deep under-barrier region, described by the $n=2,3$ eigenstates, is accompanied by the dechanneling from the nearbarrier region, which violates the exponential decay of the $n=1$ state, making effective dechanneling length (40) dependent on crystal depth. Since any redistribution of the deep under-barrier states is induced solely by the electron scattering, the depth of exponential decay law violation proves to be comparable with the electron dechanneling length, as Fig. 7 demonstrates. It also follows from the above arguments that some specific angular distribution can exist, resulting in an initial particle distribution in transverse energy $u(\xi, 0)$ so close to $u_{1}(\xi)$ as to maximally shorten the region of $u(\xi, 0)$ transformation into $u_{1}(\xi)$, making the dechanneling length notion applicable also at some $z \ll l_{\text {dech. }}$.

Thus, instead of fitting the data by the single exponential containing a constant electron dechanneling length, a more complex behavior of the dechanneling process of positively charged particles should be assumed to establish the crystal thickness most optimal for the applications. One of the most fundamental ones of the channeling in relatively thick crystals is the measurement of both magnetic and electric dipole momenta of short-living particles [7].

\subsection{Diffusion equation application to the dechanneling of negatively charged particles}

Since the particle scattering by nuclei is thoroughly taken into consideration by Eqs. (29) and (30), the solution (32) can also be applied to the case of negatively charged particles. Oppositely to the case of positively charged ones, all negatively charged particles experience strong nuclear scattering, inducing large fluctuations of transverse energy at any value of the latter. As a result, more than $50 \%$ of the particles (see Table 2 ), having transverse energies $\varepsilon_{\perp \max }<\varepsilon_{\perp} \leq V_{\max }$, experience average transverse energy variations (28) exceeding the depth $V_{\max }-\varepsilon_{\perp \max }$ of their transverse energy level occurrence. These variations induce either immediate dechanneling or large changes of both the transverse motion period and phase, making, in fact, inapplicable the whole notion of channeling, understood as a quasiperiodic transverse motion. The rest of the particles with $\varepsilon_{\perp}<\varepsilon_{\perp \max }$, which more likely can be considered as channeled, also experience large transverse energy fluctuations resulting in the uncertainty of dechanneling length reaching several tens percent-see Table 2.

Contrary to the case of positively charged particles, nuclear scattering of negatively charged ones, which occurs near the potential energy minimum, can immediately make the transverse energy of nonchanneled particles considerably less than the height of the potential barrier, giving thus rise to the intensive rechanneling process which Eqs. (29) and (30) are unable to describe. Thus, in total, the diffusion equation approach still provides merely qualitative information on the dechanneling process of negatively charged particles. Taking into consideration that the dechanneling length of negatively charged particles is much less than that of positively charged ones (see Table 2), one should acknowledge the Monte Carlo simulations to be a quite adequate approach for a study of negatively charged particle dynamics. In particular, this method correctly reproduces a nearly exponential decay of the channeling population in a bent crystal observed in [12], to describe which the diffusion equation should be additionally refined.

Monte Carlo simulations also certainly take into consideration all possible features of positively charged particle motion. In particular, they correctly describe both the rechanneling process and the large transverse energy fluctuations at the upper under-barrier region $\varepsilon_{\perp} \sim \varepsilon_{\perp \max }$, in which the diffusion equation loses its applicability. The Monte Carlo approach has also demonstrated its efficiency in a simulation of the new effects of the channeling probability increase by the crystal cut [23], both the positive miscut influence on the collimation process [24] and the other features of the latter [25], multiple new radiation features in both crystal undulators [2,26,27] and bent crystals $[28,29]$, the effects of planar channeling and quasichanneling oscillations in the deflection angle distribution of particles passed through a bent crystal [30] and many effects in the field of atomic strings [31-37]. However, the main purpose of this paper was to demonstrate that despite all the achievements of the Monte Carlo method, the diffusion equation approach, refined in the present paper, can supplement the simulation results with the enlightening treatment of the collective statistical behavior of channeling particles, which cannot be described by the sum of two exponentials decaying with nuclear and electron dechanneling lengths, respectively.

Both electron and nuclear dechanneling lengths can be measured using bent crystals [14-16]. Crystal bending will certainly change the dechanneling length values from Table 2 , evaluated for a straight one. Though both experiment and simulations demonstrate a reasonable applicability of the dechanneling length of electrons in bent crystals [12], to predict its value diffusion equation (7) should be further expanded to take into consideration the strong rechanneling effect. On the opposite, a marginal role of the rechanneling process of positively charged particles allows one to assume that the new conclusions concerning the dechanneling rate dependence on crystal depth remain true also in the presence of crystal bending and should be used to enrich the interpretation of the experiments of the type described in Ref. [14-16]. 


\section{Conclusions}

In this paper the equation of channeling particle diffusion in transverse energy was both supplemented with the effect of nuclear scattering and applied for a direct evaluation of the huge dechanneling length values at the multi- $\mathrm{TeV}$ particle energies. The diffusion equation approach has also proved to be really indispensable in revealing the general features of the collective statistical behavior of channeling particles such as the power-type channeling probability dependence on the particle penetration depths in the nuclear dechanneling region and the pronounced dependence of the effective electron dechanneling length on the particle penetration depth in the interval between the nuclear dechanneling region and approximately one electron dechanneling length. These fundamental predictions, which strongly contradict the straightforward application of both nuclear and electron dechanneling lengths, should be used for an unbiased interpretation of the simulation predictions and represent themselves a challenge for future experiments.

Open Access This article is distributed under the terms of the Creative Commons Attribution 4.0 International License (http://creativecomm ons.org/licenses/by/4.0/), which permits unrestricted use, distribution, and reproduction in any medium, provided you give appropriate credit to the original author(s) and the source, provide a link to the Creative Commons license, and indicate if changes were made.

Funded by SCOAP ${ }^{3}$.

\section{References}

1. V.G. Baryshevsky. High-Energy Nuclear Optics of Polarized Particles (World Press, Singapore, 2012)

2. V.G. Baryshevsky, V.V. Tikhomirov, Nucl. Instrum. Methods. B 309, 30 (2013)

3. A.V. Korol, A.V. Solov'yov, W. Greiner, Channeling and Radiation in Periodically Bent Crystals, Series on Atomic, Optical and Plasma Physics, vol. 69 (Springer, Berlin, 2013)

4. W. Scandale, Mod. Phys. Lett. A 27, 1230007 (2012)
5. V.M. Biryukov, Y.A. Chesnokov, V.I. Kotov, Crystal Channeling and Its Application at High-Energy Accelerators (Springer, Berlin, 2010)

6. F. Zimmermann, Nuclear Instrum. Methods B. B355, 4-10 (2015)

7. F.J. Botella et al., arXiv:1612.06769v1

8. J. Lindhard, Mat.-Fys. Medd. Dan. Vid. Selsk. 34, 1-64 (1965)

9. D.S. Gemmel, Rev. Mod. Phys. 46, 129 (1974)

10. V.V. Beloshitsky, M.A. Kumakhov, V.A. Muralev, Radiat. Eff. 20, 95-109 (1973)

11. V.V. Tikhomirov, Nucl. Instrum. Methods B36, 282-285 (1989)

12. A. Mazzolari et al., Phys. Rev. Lett. 112, 135503 (2014)

13. V.M. Biryukov et al., Nuclear Instrum. Methods B. B86, 245-250 (1994)

14. W. Scandale et al., Phys. Lett. B. 680, 129-132 (2009)

15. E. Bagli et al., Eur. Phys. J. C 74, 2740 (2014)

16. W. Scandale et al., Phys. Lett. B. 719, 70-73 (2013)

17. E.M. Lifshitz, L.P. Pitaevskii, Physical Kinetics, vol. 10 (Pergamon Press, Oxford 1981)

18. V.N. Baier, V.M. Katkov, V.M. Strakhovenko, Electromagnetic Processes at High Energies in Oriented Single Crystals (World Scientific, Singapore, 1998)

19. N.M. Matveev, Methods for integration of the ordinary differential equations (Vysshaja shkola, Moskva, 1967). (in Russian)

20. S. Pruess, C.T. Fulton, ACM Trans. Math. Softw. 19, 360-376 (1993)

21. M. Tobiyama, et al. Phys. Rev. B. 44, 9248-9258 (1991)

22. P.A. Doyle, P.S. Turner, Acta Crystallogr. 24A, 390-397 (1968)

23. V.V. Tikhomirov, JINST 2, P08006 (2007)

24. V.V. Tikhomirov, A.I. Sytov, Nucl. Phys. Invest. (Kharkov) 57, 88 $92(2012)$

25. A.I. Sytov, V.V. Tikhomirov, Nucl. Instrum. Methods. B. 355, 383386 (2015)

26. E. Bagli et al., Eur. Phys. J. C 74, 3114 (2014)

27. V.V. Tikhomirov, arXiv:1502.06588v1

28. V. Guidi, L. Bandiera, V.V. Tikhomirov, Phys. Rev. A 86, 042903 (2012)

29. L. Bandiera et al., Phys. Rev. Lett. 115, 025504 (2015)

30. A.I. Sytov et al., Eur. Phys. J. C 76, 77 (2016)

31. V.V. Tikhomirov, Phys. Lett. B. 655, 217-222 (2007)

32. W. Scandale et al., Phys. Lett. B 682, 274-277 (2009)

33. W. Scandale et al., Europhys. Lett. 93, 56002 (2011)

34. L. Bandiera et al., Nucl. Instrum. Methods. B 309, 135-140 (2013)

35. V. Guidi, A. Mazzolari, V.V. Tikhomirov, J. Appl. Phys. 107, 114908 (2010)

36. L. Bandiera et al., Phys. Rev. Lett. 111, 255502 (2013)

37. V.V. Tikhomirov, A.I. Sytov, Nucl. Instrum. Methods. B. 309, 109114 (2013) 